\title{
A Machine learning approach for the prediction of efficient iPSC modeling
}

\author{
Ravina Khandelwal ${ }^{1}$, Anuraj Nayarisseri ${ }^{*}, 1,2$
}

1. In silico Research Laboratory, Eminent Biosciences, Mahalakshmi Nagar, Indore - 452010, Madhya Pradesh, India.

2. Bioinformatics Research Laboratory, LeGene Biosciences Pvt Ltd., Indore - 452010, Madhya Pradesh, India.

\begin{abstract}
Cancer stands as major cause of mortality in the world, and it's the morbidity has significantly increased in both developing and developed nations. In spite of the recent advancement in cancer therapies, the clinical follow-up still lags far behind.Recent studies show that, stem cells are bestowed with distinctive functions, like tumor cells relocation, immunosuppression and production of bioactive elements, that helps in cancer targeting that bypassobstacles.Recent understanding show that Preclinical stem cell-based strategies has proved potential for targeted anti-tumor therapy applications. Stem cell applications in modulation and remodeling of immune system happens to be frequent procedure used past ten years in successfully treating tumor.Generation of human somatic cells into induced pluripotent stem cells (iPSCs) has often been a time consuming laborious intensive and expensive process. Additionally, the major problem with iPSCs is their tendency to revert to original somatic state. Hence, a robust computational model in discovering genes/molecules necessary for iPSC generation and maintenance can be a major leap towards in stem cell research. The synergistic combination of genetic relationship data, advanced computing hardware and nonlinear algorithms and could make artificially-induced pluripotent stem cells (aiPSC) a near future reality. Genes or proteins that are known to be essential in human pluripotent stem cells (hPSC) could possibly be used for system modelling. The present investigation is aimed to develop an unsupervised deep machine learning technology for the prediction of genes relevant in aiPSC production and its maintenance for both common and rare diseases making it a cost-effective approach.
\end{abstract}

